\title{
«¿Cuál debe ser el comienzo de la ciencia?» El problema del comienzo en Hegel
}

\author{
"With what must science begin?» \\ The problem of the beginning in Hegel
}

\author{
Nicolás GARCíA MiLls \\ Universidad de Barcelona
}

Recibido: 03-02-2011

Aceptado: 08-06-2011

\section{Resumen}

El presente trabajo se centra en el problema del comienzo de la ciencia hegeliana. Se reconstruyen los argumentos en favor de un comienzo fenomenológico y un comienzo directamente lógico a partir básicamente de una lectura del prólogo y la introducción a la Fenomenología del espiritu, por un lado, y de la introducción y el texto que abre el libro primero de la Ciencia de la lógica, por otro. La conclusión alcanzada es que el problema no sólo es un problema inherente a la noción hegeliana de ciencia filosófica, sino un problema irresoluble desde dentro del Sistema hegeliano.

Palabras clave: ciencia, comienzo, Fenomenología, Hegel, Lógica, Sistema.

\begin{abstract}
The following paper focuses on the problem of the beginning of Hegelian science. By means mainly of a reading of the preface and the introduction to the Phenomenology of spirit, on the one hand, and the introduction and the text that opens the first book of the Science of logic, on the other, the arguments in favor of a phenomenological beginning and a directly logical beginning are presented. The conclusion reached is that the problem is not only a problem inherent to the Hegelian notion of science, but one unsolvable within the Hegelian System.
\end{abstract}

Keywords: beginning, Hegel, Logic, Phenomenology, science, System. 
El interés del problema exegético en el que se centra el presente trabajo radica fundamentalmente en que se trata de un problema que afecta a la posibilidad misma de la filosofía tal y como la concibe el idealismo alemán en general y Hegel en particular. Tal concepción depende de cierta (problemática) recepción del kantismo, que le atribuye a Kant ciertas «inconsecuencias» 1 que es necesario «superar». Dichas «inconsecuencias» o tensiones que el idealismo percibe en Kant incluyen los puntos que a continuación esbozamos. (i) Las categorías sólo se pueden referir (con valor cognoscitivo) a las intuiciones sensibles. Ahora bien, todo material sensiblemente dado tiene siempre una determinada ubicación espacio-temporal (o, como mínimo, temporal), de lo cual se sigue que la razón no tiene acceso cognoscitivo a lo incondicionado-absoluto, sino sólo al ámbito fenoménico. Tal distinción entre el ámbito fenoménico y el ámbito nouménico es percibida por el idealismo ya como inconsecuencia, pues, con ella, Kant estaría poniendo como verdadero un producto de la razón que, a su vez, declara incognoscible, lo cual equivale a reconocerle a un hombre «la capacidad de tener un criterio correcto, pero con el agregado de que es incapaz de comprender nada que sea verdadero, sino sólo lo que es no verdadero. Si es absurdo eso, igualmente absurdo es un verdadero conocimiento que no conoce el objeto tal cual es en sí» ${ }^{2}$. (ii) La filosofía, en Kant, consiste en el análisis de las condiciones de la posibilidad o la forma de, en cada caso, cierto Faktum. Frente a la forma, cuya validez es a priori (y, por tanto, universal y necesaria), el contenido, esto es, aquello que debería ser lo cierto o lo válido (y cuya constitución es puesta de manifiesto por el análisis filosófico), nunca puede, por su insuperable contingencia, ser efectivamente tal, esto es, nunca puede ser objeto de una certeza absoluta. (iii) Del modo de proceder kantiano, que parte de cierto Faktum y se remonta a las condiciones de la posibilidad de ese Faktum, forma esencialmente parte el hecho de que toda dualidad o, en general, pluralidad de términos sea una pluralidad que se encuentra o constata en el curso del análisis filosófico y que es mantenida como tal pluralidad (o sea, que no se «suprime», ni se «integra», ni se «reduce», etc. en favor de una unidad «superior»). Así, tenemos una dualidad de Fakta y la correspondiente dualidad, también encontrada, de principios (apercepción pura e imperativo categórico). Y, dentro del Faktum analizado en la $\mathrm{KrV}$, tenemos, por un lado, la sensibilidad y, por otro, el entendimiento, como los dos «tron$\cos »^{3}$ del conocimiento humano (a cada uno de los cuales es, más aún, inherente también cierta pluralidad).

\footnotetext{
1 Otra cuestión es en qué medida estas «inconsecuencias» son realmente tales o, en qué medida, dentro de Kant, tienen que mantenerse. Esta cuestión (así como los desplazamientos internos a la propia obra de Kant) es, sin embargo, un problema que afecta a la lectura del conjunto de la obra crítica de Kant y que cae, por tanto, fuera de los límites temáticos del presente trabajo.

2 Hegel, G. W. F., Ciencia de la lógica. Tomo I; Trad. A. y R. Mondolfo, Buenos Aires, Solar/Hachette, 19743 , p. 44 (Gesammelte Werke, Band 21, p. 30).

${ }^{3} \mathrm{KrV}, \mathrm{A} 15 / \mathrm{B} 29$.
} 
Frente a Kant, el idealismo alemán pretende «superar» o resolver estas «inconsecuencias», exigiendo básicamente que la certeza sea una certeza absoluta, esto es, que sea, en definitiva, efectivamente tal. Esta exigencia se concreta, por de pronto, en los siguientes puntos. (i) La posición de lo incondicionado-absoluto en un «más allá» cognoscitivamente inaccesible es rechazada. Lo absoluto es el asunto mismo de la filosofía. (ii) El idealismo alemán abandona también la concepción de la filosofía como mero análisis de las condiciones de la posibilidad de un Faktum, pues de él resulta la distinción entre el ámbito fenoménico y el ámbito nouménico (con la consecuencia de que sólo podemos conocer el ámbito fenoménico, esto es, aquello que es de iure siempre contingente). La filosofía debe, pues, «deponer el nombre de amor al saber y convertirse en un saber efectivo» ${ }^{4}$. De ahí que Hegel (y en general el idealismo alemán), para referirse a la filosofía, hable frecuentemente de «ciencia(s) filosófica(s)» o sencillamente de «el saber»o «la ciencia». (iii) El modo de exposición adecuado a la filosofía (o a la ciencia filosófica o a la ciencia) en tanto que presentación de lo absoluto es el sistema en sentido fuerte. Así, encontramos reiteradamente en Hegel afirmaciones como, por ejemplo, las siguientes: «la verdadera forma en que la verdad existe no puede ser otra que el sistema científico de la verdad» ${ }^{5}$, «el saber sólo es real y sólo puede exponerse como ciencia o como sistema» ${ }^{6}$, «lo verdadero sólo es real como sistema» ${ }^{7}$, etc. Y que la filosofía debe exponerse como sistema significa justamente que debe «superarse» toda pluralidad o, lo que es lo mismo, que debe ser reducida a una pluralidad de momentos dentro de un proceso internamente necesario, pues sólo así puede quedar garantizada la completud de la exposición. La pluralidad se deduce o genera, no se encuentra o constata sin más. Cada paso en la presentación del asunto de la filosofía (esto es, de lo absoluto) tiene lugar con arreglo a un orden internamente necesario. Por tanto, todo se sigue, en último término, de un primer momento. Es precisamente en este contexto en el que el problema del comienzo de la ciencia filosófica se plantea como tal problema.

Pues bien, los términos en los que el problema aparece en la obra de Hegel son concretamente: ¿Debe el comienzo del Sistema ser un comienzo «fenomenológico»

\footnotetext{
${ }^{4}$ Hegel, G. W. F., Fenomenología del espíritu; Trad. M. Jiménez Redondo, Valencia, Pre-textos, 2006, p. 114 (Gesammelte Werke, Band 9, p. 11). En el texto al que corresponde esta cita, Hegel afirma que con la Fenomenología del espíritu pretende contribuir al acercamiento a esa meta.

5 Ibíd., p. 114 (Gesammelte Werke, Band 9, p. 11).

6 Ibíd., p. 128 (Gesammelte Werke, Band 9, p. 21).

7 Ibíd., p. 129 (Gesammelte Werke, Band 9, p. 22). En la misma línea, en el parágrafo 14 a la Enciclopedia de las ciencias filosóficas, Hegel dice: «La ciencia de éste [de lo absoluto] es esencialmente sistema». Y, en la nota a dicho parágrafo, leemos: «Un filosofar sin sistema no puede ser nada científico (...) Un contenido sólo se justifica como momento del todo; fuera de éste es una suposición infundada o certeza subjetiva» (Hegel, G. W. F., Enciclopedia de las ciencias filosóficas en compendio, Trad. R. Valls Plana, Madrid, Alianza, 20054, p. 63; Gesammelte Werke, Band 9, pp. 53-54).
} 
o debe, por el contrario, ser un comienzo «lógico»? El modo y manera como se responda a esta pregunta determina también la postura que uno vaya a adoptar ante los interrogantes que a continuación formulamos: ¿Cuál es la relación entre la Fenomenología del espíritu $(P h G)$ y la Ciencia de la lógica $(W d L)$ ? Y, más específicamente, ¿cuál es el estatuto de la $P h G$ ? Es relativamente incontrovertido que la $P h G$ es, en algún sentido, una introducción al Sistema. Pero, ¿en qué sentido exactamente? ¿Es ya la primera parte del Sistema? ¿O es una introducción meramente externa al mismo (empezando el Sistema propiamente dicho con la Lógica)?

Este conjunto de cuestiones ha sido objeto de una considerable atención dentro de la literatura secundaria. Sin embargo, no existe al respecto unanimidad alguna entre los comentaristas de Hegel, sino que cada autor adopta su particular postura interpretativa. El problema, pues, sigue abierto (y ello seguramente no es casual). En el presente trabajo pretendemos contribuir en alguna medida al debate a través de una lectura de ciertos textos hegelianos especialmente significativos a este respecto. Las tesis interpretativas que trataremos sucesivamente de defender son las siguientes: (I) La $P h G$ empieza siendo una introducción científica a (y, por lo mismo, primera parte de) el «Sistema de la ciencia». (II) A partir de la $W d L$ tiene lugar un cambio de posición o desplazamiento, que es corroborado por la formulación madura del Sistema en la Enciclopedia de las ciencias filosóficas (Enz), de acuerdo con el cual la $P h G$ es reinterpretada como una introducción meramente externa y, consiguientemente, el comienzo del Sistema propiamente dicho pasa a ser un comienzo directamente lógico. (III) El conflicto o la tensión que hay entre las posiciones presentadas en los puntos I y II respectivamente es una tensión inherente a la noción misma que Hegel tiene de la filosofía y que no puede resolverse de manera concluyente en favor exclusivamente de una $u$ otra de esas posiciones ${ }^{8}$.

\footnotetext{
8 Muestra de la mencionada falta de unanimidad la constituye Michael Forster, quien rechaza las tres tesis que acabamos de formular. Forster, en su libro Hegel's idea of a Phenomenology of spirit, defiende (en discusión con Theodor Haering, Hans-Friedrich Fulda u Otto Pöggeler entre otros) que: (i) Lo único que Hegel quiere expresar con el rótulo de «Primera parte» del Sistema bajo el cual se publicó inicialmente la $P h G$ es que la $P h G$ es una introducción que necesariamente precede al Sistema propiamente dicho. (ii) La posición del propio Hegel con respecto al lugar y función de la $P h G$ es substancialmente la misma a lo largo de toda su obra, esto es, no se produce nada parecido a una devaluación o reinterpretación de la $P h G$ por parte de Hegel en los años posteriores a su publicación. (Obviamente, si, como hace Forster, se niega que exista una tensión entre el comienzo fenomenológico y el comienzo lógico, entonces tampoco tiene ningún sentido decir que tal tensión es hegelianamente irresoluble). Sin embargo, ambas tesis, (i) y (ii), son, como trataremos de mostrar, cuando menos discutibles.
} 
En este punto presentamos la posición que Hegel asume en 1807 en relación con la cuestión de por dónde se debe empezar a filosofar, según la cual el comienzo debe ser necesariamente un comienzo «fenomenológico» (en el sentido, todavía indeterminado, específicamente hegeliano del término). En primer lugar, trataremos de exponer, en base sobre todo a la introducción a la $P h G$, en qué sentido la $P h G$ es una introducción al «Sistema de la ciencia» que es ya, ella misma, parte de la ciencia y, precisamente, primera parte de la ciencia. En segundo lugar, y partiendo del resultado entretanto alcanzado, indicaremos, en base al prólogo a la $P h G$, en qué consiste la relación entre la $P h G$ y la $W d L$ en tanto que primera y segunda parte (junto con la Filosofía de la naturaleza y la Filosofía del espíritu) del Sistema respectivamente.

La introducción a la $P h G$ se abre (párrafos 1-4) con una serie de consideraciones a propósito de la filosofía crítica (y concretamente de la idea que la filosofía crítica se hace de la naturaleza del conocimiento) y con su subsiguiente rechazo por parte de Hegel. La filosofía crítica se presenta, por de pronto, como una investigación sobre la naturaleza y los límites de la facultad de $\operatorname{conocer}^{9} \mathrm{y}$, en este sentido, constituye una consideración sobre el saber previa y distinta del saber propiamente dicho. Pero esta actitud crítica, que pretende no presuponer o aceptar nada dogmáticamente, es, en realidad, señala Hegel, la que más presupone. Sobre todo presupone que el conocimiento queda de un lado y lo absoluto de otro lado, en un «más allá» incognoscible y que, pese a quedar fuera de lo absoluto, el conocimiento es verdaderamente conocimiento ${ }^{10}$, de manera que, dice Hegel, «el miedo al error [que profesa la actitud crítica] se delata más bien como miedo a la verdad» 11 . Una crítica de la facultad de conocer, esto es, una consideración sobre el saber previa al saber o a la ciencia: (a) o bien es, en efecto, previa, es decir, externa o ajena a la ciencia, (b) o bien forma ya ella misma parte de la ciencia, con lo cual se está presuponiendo lo que con esa consideración crítica se pretende establecer o delimitar, a saber, el concepto mismo de la ciencia 12 .

\footnotetext{
${ }^{9}$ La pregunta «¿Qué puedo conocer?» expresa el primer interés de la razón ( $K r V$, A805/B833). A esa pregunta responde Kant precisamente en la $\mathrm{KrV}$.

$10 \mathrm{Y}$ esto constituye para Hegel, como hemos visto, la mayor inconsecuencia. Sólo el conocimiento de lo, digamos, verdaderamente verdadero (y no de lo de iure siempre contingente) puede ser verdaderamente conocimiento.

11 Hegel, G. W. F., Fenomenología del espíritu; op. cit., p. 181 (Gesammelte Werke, Band 9, p. 54).

12 Estas consideraciones sobre la filosofía crítica con las que se abre la introducción a la $P h G$ y que aquí hemos tratado de reproducir se encuentran resumidas por el propio Hegel en la nota al parágrafo 10 de la Enz, donde dice que: «Un punto de vista capital de la filosofía crítica consiste en que antes de emprender el conocimiento de Dios o de la esencia de las cosas es preciso investigar previamente
} 
A partir de las consideraciones que Hegel hace sobre la filosofía crítica y sobre la naturaleza del conocimiento, podemos establecer una primera (provisional) conclusión. Tal conclusión es que la ciencia debe estar presente ya desde el comienzo. Sea cual sea el comienzo, deberá ser un comienzo científico, parte de la ciencia misma. Sin embargo, que la ciencia debe estar presente ya desde el comienzo no significa, hegelianamente, que lo absoluto pueda (o incluso deba) afirmarse inmediatamente. Tal afirmación o posición inmediata de lo absoluto resulta unilateral y arbitraria.

En este punto, Hegel se separa ya, tanto de lo que él entiende o parece querer entender por «Fichte» (del yo que, al afirmarse a sí mismo inmediatamente como yo, se contrapone o enfrenta a lo otro que el yo), como de lo que él entiende o parece querer entender por «Schelling» (de la indeterminación absoluta, el $\mathrm{A}=\mathrm{A}$ o la identidad de la identidad), pues ambos autores, Fichte y Schelling, pretenden situarse de manera inmediata (como de un «pistoletazo» dice Hegel) en presencia de lo absoluto. La acusación por parte de Hegel de unilateralidad y arbitrariedad consiste, por lo que se refiere a Fichte, en la posición inmediata como absoluto de algo (a saber, el yo) que entra como término dentro de cierta contraposición y que, por tanto, se enfrenta a algo, deja algo fuera (a saber, lo otro que el yo). Pero tampoco Schelling se escapa a la acusación de unilateralidad y arbitrariedad. De hecho, es sobre todo a Schelling a quien va dirigida tal acusación. Hegel, ciertamente, acepta, como Schelling bien había visto, que, si lo absoluto tiene que ser absoluto, no puede ser esto frente a aquello o lo uno frente a lo otro, esto es, no puede ser aprehendido mediante ninguna determinación finita. Sin embargo, Schelling, con su tesis de un absoluto aconceptual o de la indeterminación absoluta, se contradice a sí mismo, puesto que, en la medida en que tal tesis se formula (se dice o se expresa de algún modo), resulta que es una determinada tesis o posición frente a otras posibles y tiene, por tanto, cierto quid o contenido. Pero entonces estamos ya en el ámbito del saber o del concepto. Y si decimos que la acusación de Hegel va dirigida sobre todo a Schelling es porque Schelling pretende escapar a la unilateralidad a costa de no observar la que, para Hegel, es la condición mínima de todo discurso filosófico, a saber, que lo absoluto, que es el asunto de la filosofía, debe decirse, debe tener una presencia conceptualmente mostrable, debe aparecer discursiva-

si la facultad de conocer es capaz de semejante tarea; hay que conocer el instrumento antes de emprender el trabajo que se debe realizar por medio de él, pues si no fuese adecuado, se emplearía vanamente el esfuerzo. Este pensamiento ha parecido tan plausible que ha provocado la mayor admiración y asentimiento y ha retrotraído el conocimiento a sí mismo, desde su interés por los objetos a la ocupación consigo, es decir, al interés por lo formal (...) Pero la investigación del conocer no puede acaecer más que conociendo. Con este, así llamado, instrumento, la investigación no significa otra cosa que conocerle y querer conocer antes de conocer es tan insensato como el sabio propósito de aprender a nadar antes de echarse al agua» (Hegel, G. W. F., Enciclopedia de las ciencias filosóficas en compendio, op. cit., pp. 111-112; Gesammelte Werke, Band 20, p. 50). 
mente. El saber (de lo) absoluto debe ser precisamente saber. Esto es algo en lo que Hegel insiste reiteradamente en el prólogo a la $P h G$. Así, por ejemplo, leemos: «La fuerza del espíritu no tiene otra magnitud que su expresión y sólo es tan grande como grande sea esa su expresión» ${ }^{13}$. Ante la aparente tensión existente entre, por un lado, el hecho de que lo absoluto no pueda ser aprehendido por medio de ninguna determinación finita (expresión en sí mismo redundante, pues toda determinación es por definición finita) y, por otro lado, el hecho de que el saber (de lo) absoluto deba ser precisamente saber, Hegel, a diferencia de Schelling, optará por la vía de intentar mostrar cómo toda determinación y contraposición tiene lugar o cae ya dentro de lo absoluto.

Retomamos, tras esta referencia a la relación (tal y como el propio Hegel parece entenderla) de Hegel con Fichte y Schelling, el hilo de nuestra exposición. La afirmación o posición inmediata de lo absoluto es, según Hegel, unilateral y arbitraria por la sencilla razón de que también el saber apareciente (das erscheinende Wissen, esto es, el saber tal y como se manifiesta, el saber inmediato, propio de la conciencia natural, el saber relativo o referido a...) se afirma como tal y toma por válidas sus propias tesis y no las de ese presunto saber absoluto. De entrada, sólo hay eso, unas tesis contra otras, todas ellas, por tanto, unilaterales y todas ellas afirmadas sin más. Y, en la medida en que sólo hay eso, a saber, unas tesis contra otras, afirmadas (como de entrada no podría ser de otra forma) sin más, todas ellas son igualmente arbitrarias, pues ninguna ha sido todavía justificada.

La ciencia, pues, necesita empezar justificando explícitamente el propio punto de vista del saber absoluto o, en otras palabras, la ciencia debe empezar por elevarse del punto de vista de la conciencia natural a la conciencia filosófica (o, como dice Hegel, al «nosotros» filosófico). Tal es la tarea de la $P h G$ y ahí radica precisamente su carácter «introductorio». Estamos ahora en condiciones de formular una segunda conclusión, a saber, que «la ciencia es ella misma fenómeno [Erscheinung]»14. La ciencia empieza teniendo lugar como exposición del saber apareciente y ello de manera que en esa exposición el saber apareciente de algún modo (en un sentido todavía por ver) se pone a prueba o se somete él mismo a examen. El comienzo de la ciencia debe ser, por tanto, fenomenológico, esto es, producirse como examen interno del saber apareciente. ¿Hasta dónde llega o se prosigue este examen del saber apareciente? Respuesta: Hasta alcanzar el punto de vista del saber absoluto.

El mecanismo de acuerdo con el cual el seguimiento del examen interno del saber apareciente conduce hasta el punto de vista del saber absoluto es esbozado por Hegel en los párrafos 6 y ss. de la introducción a la $P h G$. La $P h G$ consiste en la exposición del saber apareciente tal que en esa exposición el saber apareciente se

13 Hegel, G. W. F., Fenomenología del espiritu; op. cit., p. 118 (Gesammelte Werke, Band 9, p. 14). 14 Ibíd., p. 182 (Gesammelte Werke, Band 9, p. 55). 
examina o se pone a prueba a sí mismo. Todo examen implica algo así como un patrón o criterio (que se toma como la verdad, como la esencia o como el «en sí») y la comparación de lo que se examina con dicho criterio. Este criterio, además, no puede ser externo o traído de fuera e impuesto sobre la exposición del saber apareciente, pues ¿de dónde procedería entonces tal criterio? Sea de donde sea, en cualquier caso procedería de fuera de la ciencia. Pero si dicho criterio es externo a la ciencia, ¿qué legitimidad científica puede tener? ¿Por qué deberíamos aceptarlo como patrón o criterio de una exposición científica? Por esta vía, nos vemos devueltos, como se ve, a las aporías inherentes a la filosofía crítica que Hegel pone de manifiesto en los primeros párrafos de la introducción. El criterio, pues, debe ser, en efecto, interno al propio saber analizado. ¿Qué tipo de criterio puede ser éste? Hegel encuentra la respuesta a esta pregunta en la diferencia, inmediatamente presente a la conciencia natural, entre el «para sí̀ y el «en sí», entre el saber y la verdad o entre el concepto y el objeto.

«Esta conciencia [natural] distingue de ella algo a lo que ella a su vez está referida; o tal como ello suele expresarse también: hay algo para la conciencia; y el lado determinado de esta relación, o el lado determinado del ser de algo para una conciencia, eso es precisamente el saber. Ahora bien, de este ser-para-otro distinguimos el ser-en-sí; es decir, lo referido al saber queda asimismo distinguido de él, y queda puesto como siendo también fuera de esa relación o de esa referencia; y el lado de este en-sí es lo que se llama verdad»15.

El examen consiste, por tanto, dicho desde la perspectiva de la conciencia natural, en ver si el concepto corresponde a o concuerda con el objeto. Desde esta perspectiva, el concepto equivale al saber o al «para sí», mientras que el objeto equivale a la verdad o al «en sí». Por su parte, desde la perspectiva de la conciencia filosófica o del «nosotros», el objeto es la manifestación del saber o la figura de conciencia examinada en cada caso, mientras que el concepto es o equivale a aquello que la figura de conciencia en cuestión pone como la verdad o el «en sí»». El examen consiste entonces, dicho desde esta otra perspectiva, en ver si el objeto se corresponde con su concepto. Ambas formulaciones dicen lo mismo desde una $\mathrm{u}$ otra perspectiva ${ }^{16}$. Y el examen, formulado de un modo $u$ otro, es, en efecto, interno, puesto que la correspondencia (o la falta de ella) entre el saber y la verdad o entre el «para sí» y el «en sí» no es una cuestión que «nosotros» impongamos a la figura de conciencia examinada, sino que esa correspondencia es cuestión, tema o asunto para la propia figura de conciencia. En este sentido, dice Hegel: «En cuanto ambas cosas [saber y verdad] son para una misma conciencia, esa conciencia es ella

15 Ibíd., p. 188 (Gesammelte Werke, Band 9, p. 58).

16 Ibíd., p. 190 (Gesammelte Werke, Band 9, p. 59). 
misma la comparación de ambas; y así es para ella misma si su saber del objeto responde a ese objeto o no» 17 .

Pues bien, existen dos alternativas teóricamente posibles ante el examen al que el saber apareciente se somete a sí mismo: (a) o bien el saber y la verdad o el concepto y el objeto concuerdan y entonces todo sigue igual, (b) o bien no concuerdan y entonces surge la necesidad de modificar el saber o el concepto para que concuerde con la verdad o el objeto. La tesis de Hegel es que la alternativa que se da en cada caso (a excepción, como veremos, del último) es la (b). Cada figura resulta internamente contradictoria, se niega a sí misma o, dicho todavía en otras palabras, en cada figura resulta que hay una inadecuación entre lo que esa figura de conciencia dice hacer y lo que en realidad hace, inadecuación que, al ponerse de manifiesto, causa el hundimiento de esa figura ${ }^{18}$.

Lo que hasta aquí hemos tratado de exponer en relación con la cuestión del criterio contiene algunas implicaciones que todavía no han sido explicitadas. Es el momento de hacerlo. Por de pronto, el «en sí» resulta ser siempre el «en sí» de una determinada manifestación del saber o figura de conciencia, sólo para ella es «en sí», esto es, el «en sí» que ella pone como la verdad es en realidad $s u$ verdad, el concepto que esa figura de conciencia se hace de sí misma. Por tanto, el criterio de examen (la verdad, el «en sí») también cambia cuando aquello de lo que es criterio (la figura de conciencia correspondiente) no se sostiene en el examen, pues el «en sí» sólo para ella (para la figura de conciencia en cuestión) es «en sí», esto es, es $s u$ «en sí». Por otra parte, al reconocer que el «en sí» es siempre el «en sí» de una determinada manifestación del saber o figura de conciencia, que la verdad es $s u$ verdad o el concepto que esa figura de conciencia se hace de sí misma, etc., estamos reconociendo que la figura de conciencia en cuestión es una determinada figura de conciencia entre tantas y que de su negación (y, por tanto, de la negación de su propio criterio) surge un nuevo «en sí» y una nueva figura de conciencia que pone ese «en sí» como el «en sí» o la verdad. La negación de una figura de conciencia no es, pues, una negación abstracta, la cual tiene como resultado la nada o el puro vacío, sino que, por ser negación interna, es una negación determinada ${ }^{19}$, la cual tiene ya cierto contenido. Este contenido es la nueva figura de conciencia, que resulta de la negación interna de la figura precedente 20 .

\footnotetext{
17 Ibíd., p. 190 (Gesammelte Werke, Band 9, p. 59).

18 Hegel no puede justificar esta tesis en abstracto dentro de la introducción a la $P h G$. Por tanto, menos aún podemos tratar de dar aquí una justificación de ella. El lector debe en este punto, por así decir, tomarle la palabra a Hegel y esperar a pasar al cuerpo del texto de la $P h G$ para ver como esta tesis general se cumple en concreto, empezando por la figura de conciencia más sencilla de todas, la certeza sensible.

19 Ibíd., pp. 185-186 (Gesammelte Werke, Band 9, p. 57).

20 Este paso de una figura de conciencia a la siguiente y de esta nueva figura de conciencia, a su vez, a la siguiente, etc., es lo que Hegel llama el «movimiento dialéctico» de la conciencia o la «experien-
} 
Ahora bien, el encadenamiento de las figuras de conciencia no es percibido como tal por cada una de las figuras concretas de conciencia, las cuales ven en su propia negación simplemente su hundimiento o su muerte. Este encadenamiento internamente necesario de las figuras de conciencia, que es lo que otorga cientificidad a la exposición del saber apareciente, es, si se quiere, el único «añadido» por parte de la conciencia filosófica o del «nosotros» (esto es, de Hegel y del lector que es consciente de que de lo que se trata en todo el proceso es del saber absoluto en su generación a partir del examen del saber apareciente). Pero este «añadido» es consecuencia simplemente de la distancia, que hace posible la contemplación del proceso como tal, entre «nosotros» y la figura de conciencia analizada. Así, dice Hegel, a nosotros «no nos quedará sino puramente mirar» ${ }^{21}$.

Una vez que hemos presentado la noción de una «Fenomenología del espíritu» (o de una «Ciencia de la experiencia de la conciencia»22) tal y como aparece descrita por Hegel en la introducción a la $P h G$, podemos preguntarnos de nuevo cuál es la meta de todo el proceso o movimiento dialéctico de la conciencia y tratar de dar una respuesta que, aunque igualmente formal, resulte menos indeterminada que la que dábamos más arriba al afirmar que la meta está en la consecución del punto de vista del saber absoluto. Ahora podemos decir que la meta está fijada por el propio mecanismo que mueve el proceso y que consiste en la superación de la diferencia entre el «para sí» y el «en sí» o en la identificación del saber y la verdad, en la que el saber se desprende de su parcialidad o limitación, esto es, deja de ser uno de los términos, frente al cual hay otro, dentro de cierta contraposición. Tal es precisamente la noción de saber absoluto.

En base a la lectura que hemos realizado de la introducción a la $P h G$, podemos dar ahora la siguiente respuesta a la pregunta por el estatuto de la $P h G$. Los argumentos con los que se abre la introducción a la $P h G$ nos han llevado a concluir que la ciencia debe estar ya presente desde el comienzo. A su vez, la necesidad de jus-

cia» que la conciencia hace sobre (über) la figura de conciencia precedente. De hecho, cuando Hegel empezó a redactarla en 1805 , la $P h G$ llevaba por título "Ciencia de la experiencia de la conciencia» y en la introducción Hegel explica, en los términos que aquí hemos tratado de reproducir, el significado de este título, no el del título definitivo. Este cambio de título refleja el enriquecimiento y enorme desarrollo del proyecto inicial de la $P h G$ a lo largo de su proceso de implementación. Consideraciones de este tipo han llevado a algunos intérpretes (como Haering o Pöggeler) a denunciar el desajuste entre el plan original de la $P h G$ y su resultado, así como la falta de unidad interna a la obra. Frente a ellos, Fulda ha defendido que es esencial al proyecto de la $P h G$ el que éste se amplíe y enriquezca en su proceso de implementación y que esto no obsta para que la obra esté dotada de unidad interna. Cf.: Fulda, H. F., «Science of the phenomenology of spirit: Hegel's program and its implementation», en: Moyar, D., Quante, M. (eds.), Hegel's Phenomenology of spirit: A critical guide; Cambridge, Cambridge University Press, 2008, pp. 21-42.

${ }^{21}$ Hegel, G. W. F., Fenomenología del espiritu; op. cit., p. 190 (Gesammelte Werke, Band 9, p. 59).

22 Cf. nota 20. 
tificar explícitamente el punto de vista del saber absoluto nos ha llevado a concebir la $P h G$ como una introducción (científica) a la ciencia. Por tanto, la $P h G$ es (a) ya una determinada presentación de lo absoluto23, pero (b) una presentación tal que en ella se asume explícitamente la tarea de justificar el punto de vista del saber absoluto o el concepto mismo de la ciencia. Según esto, la $P h G$ es (a) parte del «Sistema de la ciencia» y (b) precisamente primera parte del Sistema ${ }^{24}$. El propio Hegel resume lo que hasta aquí hemos tratado de exponer en relación con el peculiar estatuto de la $P h G$ en el párrafo 27 del prólogo:

«Este devenir [Werden, llegar a ser] de la ciencia en general o del saber es lo que la presente Fenomenología del espiritu, como primera parte del Sistema, va a exponer. El saber, tal como el saber empieza siendo, o el espíritu inmediato, es lo carente de espíritu, o lo que es lo mismo: es la conciencia sensible. Para engendrar el saber propiamente dicho, o lo que es lo mismo: para engendrar el elemento de la ciencia, es decir, para engendrar lo que es el concepto puro de ésta, el espíritu tiene que trabajarse recorriendo un largo camino. -Ese devenir, tal como se articula en su propio contenido y en las figuras que se muestran en ese contenido, aparece como algo distinto [primero] de una introducción de la conciencia no científica a la ciencia; y también como algo distinto [segundo] de una fundamentación de la ciencia; $\mathrm{y}$, por supuesto, como algo completamente distinto [tercero] de esa clase de entusiasmo que empieza como con un pistoletazo con el saber absoluto, y que acaba con cualquier otro punto de vista por la decidida vía de no tomar siquiera noticia de ellos» 25 .

La $P h G$ no es, pues, ni (i) una introducción meramente externa (y por tanto acientífica) a la ciencia, ni (ii) una consideración crítica del saber previa y distinta del saber propiamente dicho (Kant), ni (iii) una afirmación o posición inmediata de lo absoluto (Fichte, Schelling), sino que es, en efecto, una introducción científica a la ciencia que es ya la primera parte de la misma.

Si la $P h G$ debe ser considerada como la primera parte del Sistema, ¿qué estatuto le corresponde a la segunda parte? ¿Y qué relación hay entre la primera y la

23 Ciertamente, que la $P h G$ es ya ella misma una determinada presentación de lo absoluto se sabe sólo al final o lo sabe en todo caso sólo la conciencia filosófica (o el «nosotros»), pero no ninguna de las figuras parciales de conciencia. De ello, esas figuras parciales de conciencia no saben nada.

${ }^{24}$ Las consideraciones que hasta aquí hemos hecho impiden concebir (como parece pretender Forster en su Hegel's idea of a Phenomenology of spirit) la cientificidad de la $P h G$ como la de una disciplina externa y distinta del Sistema propiamente dicho. Dada la noción hegeliana de la filosofía como sistema en sentido fuerte, que la $P h G$ sea una introducción científica al Sistema sólo puede significar que la $P h G$ es ya la primera parte del mismo.

25 Hegel, G. W. F. Fenomenología del espiritu; op. cit., p. 132 (Gesammelte Werke, Band 9, p. 23). En las correcciones que Hegel empezó a hacer en 1831, poco antes de morir, sobre el texto de la $P h G$ de 1807 , el fragmento que dice «aparece como algo distinto de una introducción de la conciencia no científica a la ciencia» es substituido por «va a ser algo distinto de lo que suele entenderse por una introducción de la conciencia no científica a la ciencia». 
segunda parte del «Sistema de la ciencia»? Como ya hemos mencionado, la segunda parte del Sistema está constituida por la $W d L$ (junto con la llamada Filosofía real, integrada por la Filosofía de la naturaleza y la Filosofía del espíritu). Pues bien, la $W d L$ es el despliegue o el desarrollo exhaustivo del concepto de saber absoluto alcanzado en la $P h G$. En otras palabras, la $W d L$ es la presentación de lo absoluto al margen de su surgimiento dentro del marco del examen del saber apareciente. Los distintos momentos de la presentación de lo absoluto no son ya, en la $W d L$, figuras de conciencia, sino determinaciones puras. La $P h G$ y la $W d L$ son, pues, en tanto que primera y segunda parte del «Sistema de la ciencia» respectivamente, dos modos de presentación de lo mismo, a saber, de lo absoluto. Ahora bien, en la medida en que la $W d L$ despliega o desarrolla el resultado alcanzado en la $P h G$, la $W d L$ parte de o presupone la $P h G$. Por tanto, los modos de presentación de lo absoluto son, en Hegel, necesariamente dos y necesariamente en ese orden ${ }^{26}$. Hasta aquí el planteamiento de la cuestión por parte de Hegel en 1807.

\section{II}

En el punto anterior hemos considerado los argumentos y la correspondiente evidencia textual (básicamente el prólogo y la introducción a la $P h G$ ) en favor de una lectura «fuerte» de la $P h G$, de acuerdo con la cual ésta es una introducción científica (es decir, que forma ya, ella misma, parte de la ciencia) al «Sistema de la ciencia». En este punto presentamos, en base sobre todo a la introducción a la $W d L$ y al texto que lleva por título «¿Cuál debe ser el comienzo de la ciencia?», la posición que Hegel parece asumir a partir de la $W d L$ en relación con la cuestión de por dónde se debe empezar a filosofar. Tal posición se caracteriza por una inicial continuidad con respecto a la posición de 1807 y un posterior desplazamiento en favor de un comienzo directamente lógico.

Del mismo modo que en el prólogo a la $P h G$, la $W d L$ es presentada en su introducción como el despliegue o el desarrollo exhaustivo del concepto de saber absoluto o saber puro (no relativo o referido a...) alcanzado en la $P h G$. La distinción entre el saber y la verdad, entendida ésta como algo exterior y extraño al saber, ha sido definitivamente superada a lo largo de la $P h G$. El saber absoluto (en el que el saber y la verdad han quedado igualados) constituye el elemento lógico. De este elemento ya no se sale a lo largo de todo el movimiento lógico 27 . La $W d L$, pues, parte

\footnotetext{
26 Hay varios pasajes especialmente significativos a este respecto en el prólogo a la $P h G$. Cf.: Ibíd., pp. 119, 130-131, 138, 140 (Gesammelte Werke, Band 9, pp. 15, 22-23, 28-29, 30).

27 Que el saber absoluto (en el que se ha superado la oposición entre el saber y la verdad) constituya el elemento lógico comporta que toda distinción u oposición que aparezca en la $W d L$ (por ejemplo, en la serie de parejas de términos disimétricas cuya dialéctica Hegel expone en «La doctrina de la esencia») tiene lugar dentro de ese elemento, de manera que no supone vuelta alguna al punto de vista de
} 
de o presupone el resultado de la $P h G$. Sólo porque el concepto de ciencia ya ha sido, en la $P h G$, expresamente justificado, puede, en la $W d L$, presentarse lo absoluto al margen de su surgimiento dentro del marco del examen del saber apareciente. El movimiento en el que consiste lo absoluto puede ser presentado sólo ahora, después de la $P h G$, como movimiento de las «esencialidades puras» (y no ya como movimiento de las figuras de conciencia natural). Esta relación de presuposición de la $W d L$ con respecto a la $P h G$ es expresada por Hegel en el siguiente fragmento de la introducción a la $W d L$.

«En la Fenomenología del espiritu (Bamb. y Würzb., 1807) he representado a la conciencia en su movimiento progresivo, desde su primera oposición inmediata respecto al objeto, hasta el saber absoluto. Este camino pasa a través de todas las formas de las relaciones de la conciencia con el objeto, y tiene como su resultado el concepto de la ciencia. Este concepto, pues, no precisa aquí justificación alguna (si prescindimos del hecho que surge dentro de la Lógica misma) porque ya la obtuvo en la misma Fenomenología; ni tampoco es susceptible de ninguna justificación que no sea su producción por medio de la conciencia, cuyas propias formas se resuelven todas en aquel concepto, como en su verdad (...)

El concepto de la ciencia pura y su deducción son presupuestos en el presente tratado, por cuanto la Fenomenología del espíritu no es más que la deducción de este concepto. El saber absoluto es la verdad de todas las formas de la conciencia, porque, como resultó de aquel desarrollo suyo, sólo en el saber absoluto se ha resuelto totalmente la separación entre el objeto y la certeza de sí mismo, y la verdad se igualó con esta certeza, como ésta se igualó con la verdad.

La ciencia pura presupone en consecuencia la liberación con respecto a la oposición de la conciencia. Ella contiene el pensamiento, en cuanto éste es también la cosa en sí misma, o bien contiene la cosa en sí, en cuanto ésta es también el pensamiento puro» 28 .

Así pues, según este fragmento, la ciencia hegeliana consta (o sigue constando) de dos partes, siendo la $P h G$, debido a su carácter justificatorio, necesariamente la primera parte y la $W d L$ (junto con la Filosofía de la naturaleza y la Filosofía del espíritu) la segunda. Por tanto, según el plan original de 1807, que es inicialmente asumido en la $W d L$, «debería seguir a la primera parte del Sistema de la ciencia que contiene la Fenomenología del espíritu, una segunda parte, que contuviera la Lógica y las dos ciencias reales de la filosofía, a saber, la Filosofía de la naturaleza y la Filosofía del espíritu, y así el Sistema de la ciencia estaría acabado»29.

la conciencia natural. Cf. la sección que se encuentra al final de la introducción a la $W d L$ y que lleva por título «División general de la Lógica». (Hegel G. W. F., Ciencia de la lógica. Tomo I, op. cit., pp. 54-59; Gesammelte Werke, Band 21, pp. 44- 51).

28 Ibíd., pp. 45-46 (Gesammelte Werke, Band 21, pp.32-33).

${ }^{29}$ Esta cita es del prólogo a la primera edición de la WdL. (Ibíd., p. 30; Gesammelte Werke, Band 21, p. 9). 
También en el texto titulado «¿Cuál debe ser el comienzo de la ciencia?» ${ }^{30}$, que Hegel coloca al comienzo de «La doctrina del ser», la posición acerca de la relación entre la $P h G$ y la $W d L$ sigue siendo inicialmente la misma que en 1807. Hegel afirma explícitamente al comienzo del texto que ahí se ocupará solamente del comienzo lógico, el cual presupone el resultado de la $P h G^{31}$. Ahora bien, la consideración del comienzo lógico conduce, por la naturaleza misma de la Lógica (la tensión es, pues, interna), a la consideración del comienzo sin más.

Por un lado, el carácter total y, por tanto, estrictamente sistemático de la $W d L$ parece comportar que la Lógica no pueda presuponer ninguna otra disciplina anterior. Parece, en efecto, haber una contradicción en presentar la $W d L$ (es decir, el saber absoluto «en su estado libre»o «en su medio y forma peculiar» 32 , el movimiento de las «esencialidades puras», la Filosofía especulativa o la metafísica) como resultado de otra ciencia que se encontraría «antes»o «más acá», sobre todo si se exige que la forma expositiva de la ciencia filosófica sea el sistema en sentido fuerte, el cual excluye precisamente un «antes» o un «fuera» con respecto a la ciencia. En efecto, en el sistema, cada uno de los momentos de la exposición debe seguirse del anterior con arreglo a un orden internamente necesario y, por tanto, todo se debe seguir, en último término, de un primer momento. Por otro lado, ya

30 El texto al que aquí haremos referencia corresponde a la segunda edición de la $W d L$ (de 1832). Mientras que la introducción apenas sufrió cambios en el paso de la primera a la segunda edición, el texto «¿Cuál debe ser el comienzo de la ciencia?» sí que registró modificaciones y añadidos de cierta importancia. Sin embargo, la tensión y vacilación que, como veremos, se perciben en el texto de 1832 con respecto a la posición de 1807 están ya presentes también en el texto de 1812. Los cambios que se introducen en el texto de la segunda edición van en la línea de enfatizar el desplazamiento con respecto a la posición de 1807. (Cf.: Gesammelte Werke, Band 11, pp. 33-40).

31 El siguiente fragmento no deja, a este respecto, lugar a dudas: «El comienzo es lógico, en cuanto debe efectuarse en el elemento del pensamiento libre, que existe para sí, es decir, en el puro saber. Por eso es mediato, en cuanto el puro saber es la última, absoluta verdad de la conciencia. En la introducción se observó ya que la Fenomenología del espíritu es la ciencia de la conciencia, que ella tiene por fin exponer que la conciencia tiene como resultado final el concepto de la ciencia, es decir el puro saber.

En este sentido la Lógica presupone la ciencia del espíritu fenomenológico, ciencia que contiene y demuestra la necesidad y en consecuencia la prueba de la verdad, propia del punto de vista del saber puro, y también contiene su mediación en general. En esta ciencia del espíritu en sus manifestaciones, se parte de la conciencia empírica, sensible; y ésta es el verdadero saber inmediato. En aquella misma ciencia se examinó qué contiene dicho saber inmediato. Con respecto a otras formas de conciencia, como por ejemplo, la fe en las verdades divinas, la experiencia interna, el saber por revelación interior, etc., éstas se muestran, después de breve reflexión, muy inadecuadas para ser presentadas como saber inmediato. En aquella exposición, la conciencia inmediata constituye aún lo primero y lo inmediato en la ciencia, y por tanto la presuposición; pero en la Lógica la presuposición consiste en lo que en aquella consideración se mostró como el resultado, esto es la idea como puro saber. La Lógica es la ciencia pura, es decir, el saber puro en la amplitud total de su desarrollo» (Ibíd., pp. 64-65; Gesammelte Werke, Band 21, pp. 54-55).

32 Hegel, G. W. F., Fenomenología del espíritu; op. cit., p. 183 (Gesammelte Werke, Band 9, p. 55). 
hemos señalado en qué sentido la $P h G$ y la $W d L$ (junto con la Filosofía de la naturaleza y la Filosofía del espíritu) son las dos partes del «Sistema de la ciencia». La $P h G$ y la $W d L$ son dos modos de presentación de lo mismo (a saber, de lo absoluto) y la diferencia entre un modo de presentación y otro radica en la asunción expresa por parte de la $P h G$ de la tarea de justificar el punto de vista del saber absoluto. Las dos partes de la ciencia hegeliana no son, pues, dos «mitades» cuya suma formaría un todo, sino que cada parte es ya el todo (sólo que en distintos modos de presentación en uno y otro caso). De ahí que el comienzo de cada una de las partes tienda a interpretarse como un comienzo libre de presupuestos, esto es, no como un comienzo, sino como el comienzo, sin más.

En coherencia con esto, la postura que Hegel adopta ante el problema del comienzo (y, tras las consideraciones que acabamos de hacer, podemos preguntar: ¿lógico? ¿«sólo» lógico?) es, a partir de la $W d L$, la siguiente 33 . La filosofía, entendida como sistema (en el sentido idealista), no puede empezar siendo sino una búsqueda. El comienzo es necesariamente unilateral y arbitrario y sólo a lo largo de la ulterior determinación de ese comienzo en el que consiste el movimiento total de la Lógica (o de la explicitación de lo que en ese comienzo se encontraba implícito) el comienzo se desprende de su inicial unilateralidad y arbitrariedad.

El problema de por dónde se debe empezar a filosofar sigue siendo el mismo que veíamos al comentar la introducción a la $P h G$. Por un lado, el comienzo no puede ser mediato, puesto que presupondría un «antes» o un «fuera» con respecto a la ciencia (y, por tanto, ya no sería un comienzo, sino un resultado) que, precisamente por ese carácter externo, no tendría ningún valor para la ciencia. Por otro lado, el comienzo tampoco puede ser inmediato, puesto que, al no haber sido previamente justificado, sería unilateral y arbitrario. La filosofía crítica, al anteponer al saber propiamente dicho una reflexión sobre la naturaleza y los límites del conocimiento, cae en el primer tipo de error. Tanto Fichte como Schelling (y los románticos en general), en la lectura que de ellos hace Hegel, caen, al empezar como de un «pistoletazo», en el segundo tipo de error. La solución que Hegel daba a esta disyuntiva en la $P h G$ consistía en concebir la $P h G$ como una introducción a o justificación de la ciencia que es ya, ella misma, parte (y, debido a su carácter introductorio, primera parte) de la ciencia. Por el contrario, la solución que Hegel parece dar a esta disyuntiva es ahora, una vez que la consideración del comienzo lógico ha conducido a la consideración del comienzo sin más, la siguiente. El comienzo (la identidad del ser y la nada, esto es, lo inmediato indeterminado que, precisamente por indeterminado, no excluye nada, no deja nada fuera) se justifica a sí mismo como comienzo (y justifica todo el proceso como tal) sólo al final del movimiento total de la Lógica en el que vuelve sobre sí (vuelve a la nada habiendo entretanto

33 Cf.: Hegel, G. W. F., Ciencia de la lógica. Tomo I; op. cit., p. 66 y ss. (Gesammelte Werke, Band 21, pp. 56 y ss.). 
generado y suprimido toda determinación, esto es, vuelve sobre sí como lo mediato absoluto) y muestra así estar a la altura de lo que se exige del comienzo de la filosofía o la ciencia en tanto que presentación de lo absoluto. En el comienzo debe estar ya, en efecto, todo, de modo que a lo largo de todo el proceso no se sale del comienzo ni se transita a algo verdaderamente distinto ${ }^{34}$. Todo el desarrollo ulterior no es sino una ulterior determinación o explicitación de lo que ya hay al comienzo. Si lo absoluto ha de ser absoluto no puede ser esto frente a aquello o lo uno frente a lo otro, esto es, no puede ser nada determinado, sino que habrá de ser la vaciedad o la indeterminación absoluta. Ahora bien, en lo absoluto debe tener lugar precisamente todo, toda determinación. Por tanto, la presentación de lo absoluto no puede consistir, como en Schelling (leído por Hegel), pura y simplemente en negar toda determinación y remitir a una «niebla» o a «la noche en la que todos los gatos son pardos» 35 , sino que habrá de mostrarse cómo, a partir de esa inicial vaciedad, se genera todo, toda determinación 36 . La conclusión que podemos sacar del aparente desplazamiento o viraje con respecto a la posición de 1807 es que la justificación o la mostración de la no unilateralidad del punto de vista del saber absoluto ya no corresponde (o no tiene por qué corresponder) a la $P h G$, sino que corresponde a la propia $W d L^{37}$.

34 Cf. nota 27.

35 Hegel, G. W. F., Fenomenología del espíritu; op. cit., p. 122 (Gesammelte Werke, Band 9, p. 17).

${ }^{36}$ Quizá sea conveniente indicar algo acerca de la relación entre la posición de Hegel y la posición de Reinhold según la cual el comienzo debe ser un comienzo «hipotético» o «problemático». De entrada, Hegel reconoce en «¿Cuál debe ser el comienzo de la ciencia?» que hay parte de verdad en la propuesta de Reinhold. Y, de hecho, más allá de la crítica de Hegel a Reinhold, parece haber ciertas similitudes entre ambos en lo que a la cuestión del comienzo se refiere. Ciertamente, en Hegel, el comienzo no puede ser cualquier cosa, sino que deberá ser lo inmediato indeterminado, esto es, el puro ser. Sólo el puro ser puede valer como el primer momento de una presentación sistemática de las determinaciones del pensamiento puro, pues todas las demás determinaciones presuponen alguna otra determinación previa. En este sentido, difícilmente puede decirse que el comienzo sea, en Hegel, un comienzo meramente «hipotético» o «problemático». Ahora bien, como acabamos de decir en el cuerpo del texto, ese mismo comienzo, el puro ser, sólo se justifica como tal cuando se logra mostrar que en él está ya todo (o que de él se sigue todo), es decir, cuando se vuelve a lo que había al comienzo, pero habiendo entretanto generado y suprimido toda determinación. Y en esto sí que parece haber cierta similitud entre la propuesta de Reinhold y Hegel.

37 En coherencia con el desplazamiento que se produce en la $W d L$ con respecto a la $P h G$, la posición de Hegel en relación con el problema de la intuición intelectual también parece variar. La $P h G$ pretendía mostrar que la intuición intelectual es, no sólo innecesaria, sino ilegítima. Ahora, sin embargo, la cuestión no es si puede (o no) haber una intuición intelectual o algo parecido, sino si el contenido de esa intuición es (o no) un contenido concreto o determinado. Y el problema es que el yo que Reinhold y Fichte afirman inmediatamente, precisamente por ser afirmado inmediatamente, no puede ser sino el yo de la conciencia natural o empírica, cuya parcialidad o limitación, esto es, cuya contraposición a otro término, a saber, a la verdad o el objeto, es lo que el saber absoluto exige que se haya superado. El único, digamos, contenido intuicional que puede haber al comienzo es el puro ser, la nada, el vacío lógico. Cf.: Hegel, G. W. F., Ciencia de la lógica. Tomo I; op. cit., pp. 70-72 (Gesammelte Werke, Band 21, pp. 62- 65). 
Este desplazamiento en favor de un comienzo puramente lógico queda corroborado tanto por la formulación madura del Sistema en la $E n z$, como por la nota del año 1831 que Hegel añade al prólogo a la primera edición de la $W d L$. En la Enz el Sistema empieza directamente por la Lógica ${ }^{38}$, de manera que la $P h G$ queda destituida de su carácter de introducción científica al Sistema. ¿Qué ocurre entonces con la $P h G$ ? Respuesta: Parte del contenido de la $P h G$ queda incluido dentro de la Filosofía real como segundo momento de la Filosofía del espíritu subjetivo, pasando así a ser considerado como una mera aplicación del «método» de la Lógica a un objeto particular (a saber, a la conciencia empírica). La $P h G$, como primera parte de la ciencia, es expulsada del Sistema y queda relegada al estatuto de una introducción meramente externa desprovista de valor científico (aunque quizá no de algún tipo de valor didáctico o propedéutico) ${ }^{39}$. La nota del año 1831 al prólogo a la primera edición de la $W d L$ va en la misma línea. En ella, Hegel dice: «Este título [el de «Primera parte» del «Sistema de la ciencia»] ya no figurará más en la segunda edición [de la $P h G$ ], que aparecerá para las próximas Pascuas. En lugar de la segunda parte que me proponía escribir (...) y que debía contener el conjunto de las otras ciencias filosóficas, he publicado, desde entonces, la Enciclopedia de la ciencias filosóficas, cuya tercera edición salió el año pasado» 40 .

Sin embargo, estas modificaciones en la formulación madura del Sistema con respecto a la formulación de 1807 no cuentan de por sí como argumentos en favor de la posición según la cual el comienzo debe ser un comienzo directamente lógico (y, en consecuencia, en contra de una lectura «fuerte» de la $P h G$ ), sino que simplemente constituyen una expresión del problema del difícil encaje de la $P h G$ y la $W d L$. ¿Qué motivos pueden darse entonces en favor del desplazamiento intrasistemático según el cual el comienzo debe ser un comienzo lógico? Por de pronto, podemos hacer las siguientes consideraciones al respecto, las cuales pretenden resumir parte de lo que hemos tratado de exponer en este punto.

(i) El «método» de la Lógica debe ser un método estrictamente sistemático, es decir, tal que todo se siga de un primer momento con arreglo a un orden internamente necesario, quedando así garantizada la completud del proceso. Esta misma exigencia de completud comporta que el comienzo lógico debe ser un comienzo radical, esto es, libre de presupuestos. Pues bien, ante esta imposibilidad de presuponer un «fuera» o un «antes» con respecto al movimiento lógico, la $P h G$ pasaría a ser considerada como una reflexión previa externa al Sistema propiamente dicho.

\footnotetext{
38 Ciertamente, a la versión de la Lógica contenida en la Enz, Hegel antepone una extensa sección que lleva por título «Concepto previo [Vorbegriff]». Sin embargo, ni la introducción a la Enz ni dicha sección cumplen la función de una introducción científica a la ciencia. Ambos textos tienen un carácter declaradamente «histórico», es decir, acientífico y son, por tanto, externos al Sistema propiamente dicho. El sistema sensu stricto empieza, sin más, por la Lógica.

$39 \mathrm{Cf}$., sin embargo, el punto III.3.

40 Hegel, G. W. F., Ciencia de la lógica. Tomo I; op. cit., p. 30 (Gesammelte Werke, Band 21, p. 9).
} 
(ii) El puro ser, lo inmediato indeterminado es, en cierto modo paradójicamente, necesariamente unilateral y arbitrario y sólo al final de su propio desarrollo, en el curso del cual se genera y suprime toda determinación, se desprende de su inicial unilateralidad y resulta ser lo mediato absoluto. Sólo al final, el comienzo muestra estar a la altura de lo exigido de él (a saber, de la exigencia de que de él se siga toda determinación), justificándose como comienzo y justificando todo el proceso como tal. La $W d L$ tiene, pues, un carácter autojustificatorio. El movimiento lógico legitima el propio punto de vista especulativo o lógico. De acuerdo con esto, la justificación de la perspectiva absoluta ya no pertenecería (o no tendría por qué pertenecer) a la $P h G^{41}$.

(iii) Hegel, tanto en la $P h G$ como en la $W d L$, pretende distanciarse de cualesquiera posiciones «subjetivistas» según las cuales el comienzo radica en la intuición intelectual del yo o en la certeza de sí mismo, pues el yo se define por su oposición al objeto y, por tanto, comporta siempre cierta parcialidad o limitación. Pues bien, el Hegel especulativo o lógico parece ver cierta proximidad, que es necesario eliminar, entre la $P h G$ y tales posiciones «subjetivistas». Los momentos de la presentación de lo absoluto no pueden ser figuras de conciencia, sino que deben ser determinaciones puras, en las que se ha superado la oposición inherente a la conciencia natural ${ }^{42}$.

Hasta aquí la reformulación (inicial continuidad y posterior desplazamiento con respecto a la posición de 1807) del problema del comienzo en la $W d L$.

\section{III}

En este último punto aportamos nueva evidencia textual y presentamos algunas ulteriores consideraciones en favor de una lectura «fuerte» (primera parte del «Sistema de la ciencia») y una lectura «débil» (introducción externa al Sistema) de la $P h G$ (puntos I y II respectivamente) y discutimos la consistencia del desplazamiento intrasistemático de acuerdo con el cual el comienzo debe ser un comienzo, sin más, lógico (punto II). Empezamos, en III.1, cuestionando si las tres consideraciones, (i), (ii) y (iii), con las que cerrábamos el punto anterior constituyen motivos suficientes para un cambio de posición por lo que a la pregunta por el comienzo del Sistema se refiere, reivindicando así el lugar y el estatuto de la $P h G$. En III.2 pre-

\footnotetext{
41 Ya en un fragmento de la introducción a la $W d L$ anteriormente citado en el cuerpo del texto aparecía en este sentido una afirmación, contenida entre paréntesis, un tanto desconcertante. El fragmento y la afirmación son los siguientes: «Este concepto [el concepto de la ciencia], pues, no precisa aquí justificación alguna (si prescindimos del hecho que surge en la Lógica misma) porque ya lo obtuvo en la misma Fenomenología» (Ibíd., p. 46; Gesammelte Werke, Band 21, p. 32). Las cursivas son mías. 42 Cf. en especial: Ibíd., pp. 45 y 71 (Gesammelte Werke, Band 21, pp. 31 y 63-64).
} 
sentamos algunas consideraciones que, de ser acertadas, mostrarían que la $P h G$ no cumple su pretendida función de ser una introducción científica al punto de vista del saber absoluto a partir de la conciencia natural y que, por tanto, hablarían en favor de la posición según la cual el comienzo debe ser un comienzo directamente lógico. Por último, en III.3, sugerimos, dada la presencia de buenos argumentos y amplia evidencia textual en favor tanto de una como de otra alternativa (lectura «fuerte» y lectura «débil» de la $P h G$ ), que el problema es un problema no sólo inherente a la propia noción hegeliana de ciencia, sino hegelianamente irresoluble.

1. Ya hemos señalado cómo la reubicación de parte del contenido de la $P h G$ en la $E n z$ y la nota que Hegel añade en 1831 al prólogo a la primera edición de la $W d L$ no cuentan de por sí como argumentos en favor de la posición según la cual el comienzo debe ser un comienzo lógico, sino que simplemente señalan la dificultad que tiene Hegel para encajar la $P h G$ dentro del Sistema en su formulación madura y constituyen, por tanto, una expresión del problema mismo. De hecho, ni la reformulación del Sistema en la $E n z$ ni la nota del año 1831 niegan el fondo de la cuestión, a saber, que la $P h G$ tiene el mismo carácter total que la $W d L$, consistiendo la diferencia entre ambas en el carácter, a su vez, «introductorio» de la $P h G$. Más aún, al menos dos pasajes de la $E n z$ (pertenecientes a la introducción y a la sección que lleva por título «Concepto previo» respectivamente) pueden leerse como una reivindicación de la función y el estatuto de la $P h G$. En primer lugar, en el parágrafo 12, pese a que no se menciona expresamente la $P h G$, Hegel describe un proceso de ascensión hasta el punto de vista del saber absoluto muy similar (incluso terminológicamente) a aquél llevado a cabo en la $P h G^{43}$. Y, en segundo lugar, en la nota al parágrafo 25, Hegel aclara por qué y en qué sentido la primera edición de la $P h G$ llevaba el título de «Primera parte» del «Sistema de la ciencia» y cómo «lo que pertenece a las partes concretas [de la filosofía] coincide ya parcialmente con aquella introducción». Frente a la $P h G$, entendida como introducción científica, Hegel dice que: «La consideración preliminar que hacemos aquí [con la consideración de los tres posicionamientos del pensamiento respecto de la objetividad] tiene más aún la incomodidad de poderse hacer solamente de manera histórica y raciocinante [räsonnierend] $\rangle^{44}$, esto es, acientífica.

Estos dos pasajes parecen hablar en contra de un comienzo directamente lógico. A través de las siguientes preguntas pretendemos cuestionar (o, cuando menos, expresar algunas dudas en relación con) las consideraciones (i), (ii) y (iii) con las

43 Cf.: Hegel, G. W. F., Enciclopedia de las ciencias filosóficas en compendio; op. cit., pp. 113-114 (Gesammelte Werke, Band 20, p. 52).

44 Ibid., p. 132 (Gesammelte Werke, Band 20, p. 69). El «más aún» posiblemente haga referencia a defectos en la ejecución del plan de la $P h G$ (concretamente del capítulo $\mathrm{V}$ en adelante), de los que Hegel era, ciertamente, consciente y de los que ya se quejaba a Niethammer y a Schelling en cartas de Enero y Mayo de 1807 respectivamente. 
que cerrábamos el punto anterior y con las que pretendíamos resumir los motivos del cambio de posición en favor de un comienzo directamente lógico, reivindicando así, en la misma línea que los pasajes a los que nos acabamos de referir, la función y el estatuto científico de la $P h G$. Las consideraciones, en la forma de preguntas retóricas, en favor de un comienzo fenomenológico, esto es, de una lectura «fuerte» de la $P h G$ son las siguientes.

(i) ¿En qué medida puede el elemento lógico como tal, como plano o punto de vista, autojustificarse a lo largo de su propio desarrollo (aún siendo toda especulación una búsqueda, de manera que sólo al final el comienzo y el entero proceso se justifican como tales, etc.)?

(ii) ¿En qué medida, por tanto, el elemento lógico, considerado separadamente, prescindiendo totalmente de la $P h G$, no es unilateral y arbitrario, pudiendo entonces uno hacerle a Hegel los mismos reproches que a quienes pretenden hallarse inmediatamente, como de un «pistoletazo», en presencia de lo absoluto (aún siendo lo absoluto su propio movimiento o desarrollo, de manera que sólo al final de tal desarrollo lo absoluto es propiamente lo que es, etc.)?

(iii) ¿En qué medida es posible una superación o un completo distanciamiento con respecto a toda posición «subjetivista» (posición que pone el comienzo en el yo o en la certeza de sí mismo)? ¿No se trata en todo esto precisamente de saber absoluto? «Yo» $\mathrm{o}$ «saber» aparecen, de entrada, como términos de una contraposición y comportan, por tanto, cierta limitación o parcialidad. Pero la $P h G$ constituye justamente el camino por el que el yo o el saber superan cualquier oposición con respecto al objeto o la verdad (camino en el que, ciertamente, tales términos, «yo» o «saber», se vuelven acaso en alguna medida incómodos o ambiguos, pues señalan, de entrada, un enfrentamiento y una limitación, que es precisamente lo que se ha superado).

Estas tres preguntas pretenden, todas ellas, señalar una misma sospecha o reserva. Tal sospecha es que el punto de vista del saber absoluto, inmediatamente afirmado, resulta unilateral y arbitrario. La conciencia natural o inmediata no puede (ni tiene por qué poder) situarse, ya de entrada, en presencia de lo absoluto, ni tiene por qué aceptar ese saber que se declara a sí mismo absoluto. Lo verdaderamente inmediato no es el puro ser del comienzo de la $W d L$, sino el saber que profesa la conciencia natural. Que la filosofía debe ser la presentación de lo absoluto sólo se justifica por medio de la mostración del carácter internamente contradictorio (y del necesario encadenamiento $)^{45}$ de todas las figuras de conciencia a excepción de la última, constituida por el propio saber absoluto (que supera y a la vez integra todas las anteriores figuras parciales de conciencia). $\mathrm{Y}$ esa es precisamente la tarea que Hegel acomete en la $P h G$.

\footnotetext{
45 Sin esa necesidad no se podría tener la certeza de que las figuras de conciencia expuestas son, en efecto, todas las figuras de conciencia posibles, con lo cual cabría la posibilidad de que existiese alguna otra figura (distinta del saber absoluto) que no resultase ser internamente contradictoria.
} 
2. Pese a las reservas que acabamos de expresar y a los pasajes de la introducción a la $E n z$ y la sección que lleva por título «Concepto previo» que hemos citado, no deja de ser cierto que, en su formulación madura en la Enz, el Sistema hegeliano empieza por la Lógica (a la que siguen la Filosofía de la naturaleza y la Filosofía del espíritu). Esto, además, es así, según Hegel, no por ninguna imposición externa, sino por la propia naturaleza o la estructura interna de lo absoluto. Consiguientemente, la $P h G$ queda destituida de su carácter de introducción científica al (y primera parte del) Sistema, quedando su contenido parcialmente incluido dentro de la $E n z$, pero no como introducción científica, sino como segundo momento de la Filosofía del espíritu subjetivo.

A esta consideración general referente a la arquitectura del Sistema tal y como es expuesto en la $E n z$ hay que añadir los diversos pasajes de la introducción a la Enz y el «Concepto previo» que confirman el cambio de posición que hemos presentado en II. Para empezar, ya al comienzo del prólogo a la primera edición (1817) Hegel afirma que la $E n z$ constituye una «visión de conjunto del ámbito total de la filosofía» 46 . Más adelante, en el parágrafo 17 de la introducción a la Enz, encontramos una reflexión en torno a la cuestión del comienzo que coincide exactamente con la posición que, a través básicamente de una lectura del texto que lleva por título «¿Cuál debe ser el comienzo de la ciencia?», hemos identificado como la posición que Hegel oficialmente adopta a partir de la $W d L^{47}$. Por último, en la nota al parágrafo 78 de la $E n z$, Hegel rechaza el escepticismo como posible introducción a la Lógica (y recuérdese que en el párrafo 6 de la introducción a la $P h G$ ésta era caracterizada como «el escepticismo cumpliéndose») 48 en favor simplemente de «la decisión libre de querer pensar con toda pureza» 49 .

\footnotetext{
46 Ibíd., p. 81 (Gesammelte Werke, Band 20, p. 23).

47 Cf.: Ibíd., pp. 119-120 (Gesammelte Werke, Band 20, p. 59).

48 Hegel, G. W. F., Fenomenología del espíritu; op. cit., p. 184 (Gesammelte Werke, Band 9, p. 56). Esta expresión aparece precedida de otra, de acuerdo con la cual la $P h G$, vista desde la perspectiva de la conciencia natural, puede considerarse «como el camino de la duda [Zweifel] o, más propiamente, como el camino de la desesperación [Verzweiflung]» (Ibíd., p. 184; Gesammelte Werke, Band 9, p. 56). Que ambas expresiones aparezcan juntas se entiende por cuanto el escepticismo moderno se basa en la incertidumbre o la duda con respecto a la correspondencia (o falta de correspondencia) entre el saber y su objeto. Por tanto, la posibilidad misma de la duda escéptica descansa sobre la distinción entre el saber y el objeto inherente a la conciencia natural. La $P h G$ es el examen y seguimiento de las diversas formas que adopta esta distinción (esto es, de las diversas figuras de conciencia) y la mostración de que en toda figura, a excepción de la última, hay una inadecuación o contradicción interna. Sólo en el saber absoluto, al quedar identificados ambos términos, se ha superado la contraposición entre saber y verdad. Hegel, por tanto, no trata de esquivar o evitar el reto escéptico, sino que lo asume y, a lo largo de la $P h G$, se enfrenta a y destruye la posibilidad misma del escepticismo. Es justamente en este sentido en el que puede decirse que la $P h G$ es «el escepticismo cumpliéndose».

49 Hegel, G. W. F., Enciclopedia de las ciencias filosóficas en compendio; op. cit., p. 182 (Gesammelte Werke, Band 20, pp. 117-118). Cf. también el parágrafo 36 de la primera edición de la Enz (Gesammelte Werke, Band 13, pp. 43-35).
} 
Además de ofrecer esta evidencia textual, podemos hacer todavía las siguientes consideraciones en favor de un comienzo lógico, esto es, de una lectura «débil» de la $P h G^{50}$.

(i) En primer lugar, cabe reconocer en la $P h G$ la persistencia de la necesidad de algo así como una intuición intelectual o cierta presencia inmediata de lo absoluto. En efecto, asumir las manifestaciones del saber o las figuras de conciencia sin recortarlas o considerarlas de manera abstracta, ¿no presupone una previa noción de qué es lo que implica una tal presencia sin recortes, esto es, de la distancia entre la conciencia natural y la conciencia filosófica (o el «nosotros») que se encuentra ya en el punto de vista del saber absoluto? Si esto es así, la $P h G$ no haría innecesaria o mostraría la inadecuación de una intuición intelectual o un acceso inmediato a lo absoluto, sino que los seguiría presuponiendo. Por tanto, la $P h G$ no cumpliría su función de ser una introducción científica a la perspectiva filosófica a partir de la conciencia natural.

(ii) Ya hemos visto cómo la $P h G$ y la $W d L$, en tanto que primera y segunda parte del «Sistema de la ciencia», no deben ser entendidas como dos «mitades» meramente yuxtapuestas la una a la otra, sino que son dos modos de presentación de lo mismo, a saber, de lo absoluto. Debe existir, pues, algo así como una correspondencia entre la $P h G$ y la $W d L$. Hegel incluso afirma en algún lugar que existe (o debe existir) una correspondencia biunívoca entre las figuras de conciencia de la $P h G$ y las categorías de la $W d L$ y, más aún, que las figuras de conciencia de la $P h G$ descansan sobre o presuponen las categorías de la $W d L$ (o de alguna otra versión anterior de la Lógica) ${ }^{51}$. Según esto, lo que, por así decir, queda del tránsito de una figura de conciencia a la siguiente, si se elimina su carácter fenomenológico o «existencial», es la dialéctica puramente lógica, el movimiento de las «esencialidades puras». Y es sólo desde esta Lógica subyacente que es posible percibir la necesidad en el paso de una figura de conciencia a la siguiente. Por ejemplo, sólo desde

\footnotetext{
50 Los problemas para una lectura «fuerte» de la $P h G$ que a continuación señalamos se encontraban, cuando menos implícitamente, ya presentes en la exposición que hemos hecho en I. (Cf. en especial la nota 23, así como las referencias que ahí hacíamos al «nosotros» y a la cientificidad de la $P h G$ ).

51 El problema de la correspondencia entre las figuras de conciencia de la $P h G$ y las categorías de (alguna versión de) la Lógica es una cuestión sobre la que se ha debatido bastante en la literatura secundaria sobre Hegel. Cf. por ejemplo: Forster, M. Hegel's idea of a Phenomenology of spirit; Chicago, The University of Chicago Press, 1998, pp. 501-543 (y, en especial, pp. 511-535). Ahí, Forster discute básicamente con Fulda (y también con Heinrichs). Según Fulda (en su artículo «Zur Logik der Phänomenologie von 1807» contenido en el libro Materialien zu Hegels «Phänomenologie des Geistes»), la versión de la Lógica que subyace a la $P h G$ debe buscarse en el breve borrador de Filosofía especulativa que se encuentra hacia el final de la Filosofía del espíritu del año 1805-1806. Forster, por el contrario, sostiene que la versión de la Lógica que subyace a la $P h G$ se encuentra en una temprana versión de la Enciclopedia del año 1808. (Por lo demás, Forster considera que esta dependencia con respecto a la Lógica es un defecto en la ejecución del plan de la $P h G$ y no un rasgo definitorio del mismo).
} 
esta Lógica subyacente sería posible percibir cómo de la negación determinada (esto es, negación que es a la vez el cumplimiento o la realización de eso mismo que se niega) de la certeza sensible se genera necesariamente la percepción, cómo de la negación determinada de la percepción se genera necesariamente el entendimiento y cómo a partir de la negación determinada del entendimiento se supera el ámbito de la conciencia para pasar a la esfera de la autoconciencia.

Ya hemos expuesto (en I y II) en qué sentido la $W d L$ parte de y por tanto presupone el resultado de la $P h G$. Pues bien, ahora nos encontramos con que la $P h G$ presupone (depende de, se funda en, etc.), a su vez, alguna versión de la Lógica. Algunos de los textos en los que Hegel afirma la existencia de una correspondencia entre la $P h G$ y la Lógica y la presuposición de las categorías de la Lógica por parte de las figuras de conciencia de la $P h G$ son los siguientes. En primer lugar, en el párrafo 34 del prólogo a la $P h G$, Hegel dice:

«Este movimiento de las esencialidades puras [esto es, la Lógica] constituye la naturaleza de la cientificidad en general. Considerada en lo que respecta a la textura [Zusammenhang] de su contenido, esa cientificidad es la necesidad y el extenderse de ese contenido, por los que ese contenido acaba transformándose en un todo orgánico. El camino por el cual se alcanza el concepto del saber [esto es, la $P h G$ misma] se convierte igualmente $a$ través de esa cientificidad en un devenir necesario y completo» 52 .

En segundo lugar, casi al final del capítulo VIII de la $P h G$, leemos:

«(...) a cada momento abstracto de la ciencia corresponde una forma o figura del espíritu apareciente. Y así como el espíritu apareciente, es decir, el espíritu en su aparecer ahí o en su estar ahí [der daseiende Geist], no es más rico que ella [que la ciencia], tampoco él es más pobre que ella en su contenido» ${ }^{53}$.

Por último, en el prólogo a la primera edición de la $W d L$, Hegel afirma:

«La conciencia es el espíritu como conocimiento concreto y circunscrito en la exterioridad; pero el movimiento progresivo de este objeto, tal como el desarrollo de toda la vida natural y espiritual, sólo se funda en la naturaleza de las puras esencias, que constituyen el contenido de la Lógica» ${ }^{54}$.

\footnotetext{
52 Hegel, G. W. F., Fenomenología del espiritu; op. cit., p. 138 (Gesammelte Werke, Band 9, pp. 2829). Las cursivas son mías.

53 Ibíd., p. 911 (Gesammelte Werke, Band 9, p. 432).

${ }^{54}$ Hegel, G. W. F., Ciencia de la lógica. Tomo I; op. cit., p. 30 (Gesammelte Werke, Band 21, p. 8).

Cf. también el fragmento citado en la nota 41.
} 
Ahora bien, si, como estos textos indican, la $P h G$ presupone alguna Lógica subyacente, entonces resulta que Hegel alcanza la perspectiva del saber absoluto, esto es, el elemento lógico, sólo porque ya lo estaba (más o menos tácitamente) presuponiendo y estaba desde el principio en él. Si esto es así, la «Fenomenología del espíritu» (o la «Ciencia de la experiencia de la conciencia») no sería una disciplina científica independiente o autónoma, sino un caso particular de aplicación de las categorías de la Lógica. (En efecto, para que la $P h G$ fuese una disciplina científica independiente, debería consistir en la elevación a la perspectiva del saber absoluto a partir de la conciencia natural y, por tanto, no presuponer información alguna de la ciencia o la Lógica misma, sino que debería desarrollar la dialéctica de las figuras de conciencia de manera inmanente a las propias figuras analizadas). Por tanto, la $P h G$ no sería en realidad una introducción científica a (y primera parte de) la ciencia, sino en todo caso una introducción externa al Sistema propiamente dicho ${ }^{55}$.

Si estas consideraciones, (i) y (ii), son acertadas, entonces parecería que, en efecto, la $P h G$ fracasaría en su propósito original de ser una introducción científica a la ciencia a partir de la conciencia natural, debiendo ser entonces el comienzo un comienzo directamente lógico, en cuyo elemento Hegel (y el «nosotros» de la $P h G$ ) ya desde el principio se encontraría. La conciencia natural y el lector todavía no familiarizado con la ciencia hegeliana sencillamente no podrían entender la $P h G$ y, por tanto, serían incapaces de ascender, con ayuda de la $P h G$, a la perspectiva científica a no ser que ya se encontrasen en ella desde el principio56. Pero, en caso de

55 Esta dificultad para una lectura «fuerte» de la $P h G$ es detectada ya por Fulda en su libro Das Problem einer Einleitung in Hegels Wissenschaft der Logik. La posición de Fulda con respecto al lugar y estatuto de la $P h G$ es, muy resumidamente, la siguiente. Según Fulda, Hegel inicialmente concibió la $P h G$ como una introducción científica a (y primera parte de) su «Sistema de la ciencia», la cual, en tanto que disciplina científica independiente, no incluía presuposición alguna del resto del Sistema (y en concreto de la Lógica). Sin embargo, ya desde el principio, la $P h G$ sí que se basa de hecho en cierta versión de la Lógica, contradiciendo así su intención original. Esto llevó finalmente a Hegel a reinterpretar la $P h G$, pasando a considerarla, no ya la primera parte de la ciencia, sino una introducción a la ciencia hegeliana basada en cierta presuposición de la ciencia misma. Cf.: Fulda, H. F., Das Problem einer Einleitung in Hegels Wissenschaft der Logik; Frankfurt am Main, Vittorio Klostermann, 1965, pp. 79-115. Esta posición es compartida por Jürgen Habermas, quien en el punto I.1 de Erkenntnis und Interesse, dice: «Hegel hatte zunächst die «Phänomenologie» als ersten Teil des Systems der Wissenschaft publiziert. Er war damals der Überzeugung, daß die Gestalten des erscheinenden Bewußtseins mit Notwendigkeit einander folgen, und «durch diese Notwendigkeit ist dieser Weg zur Wissenschaft selbst schon Wissenschaft». Indessen konnte Hegel eine Notwendigkeit im Fortgang der phänomenologischen Erfahrung erst vom Standpunkt absoluten Wissens rückblickend in Anspruch nehmen (...) Unter diesem Gesichtspunkt würde aber die phänomenologische Untersuchung ihr Spezifisches einbüßen und auf die Ebene einer Realphilosophie des Geistes herabgesetzt» (Habermas, J., Erkenntnis und Interesse; Frankfurt am Main, Suhrkamp, 1968, pp. 32-33). Forster, por el contrario, rechaza la tesis general de que Hegel sometió la $P h G$ a una reinterpretación fundamental o devaluación después de su publicación. Para una discusión al respecto tanto con Fulda como con Haering y Pöggeler (quienes también afirman que sí tiene lugar una tal reinterpretación y devaluación), cf.: Forster, M., Hegel's idea of a Phenomenology of spirit; op. cit., pp. 547-570. 
encontrarse ya en la perspectiva científica, ¿para qué necesitarían una introducción en la que se describiese el proceso de ascensión hasta dicha perspectiva?

3. El seguimiento que hemos realizado de los textos de Hegel en I y II (junto con las consideraciones que hemos hecho en III.1 y III.2) muestra que existen buenos argumentos y amplia evidencia textual en favor tanto de una como de otra de las alternativas presentadas. Ambas alternativas, no obstante, tienen sus respectivos inconvenientes y deben hacer frente a serias dificultades. La lectura «fuerte» de la $P h G$ (I y III.1) se basa sobre todo en la necesidad de una introducción explícita (que, para poder ser tomada en consideración por la ciencia, tiene que ser ella misma científica) al saber absoluto, pues, de lo contrario, el punto de vista del saber absoluto resultaría tan unilateral y arbitrario como cualquier conjunto de tesis inmediatamente afirmadas que se pretendieran tener por válidas por el mero hecho de que son así afirmadas. Sin embargo, esa introducción resulta que sólo se puede llevar a cabo presuponiendo desde el principio la propia perspectiva científica o el elemento lógico, con lo cual dicha introducción se convierte en redundante y, por tanto, superflua. El comienzo de la ciencia debe ser, en consecuencia, un comienzo directamente lógico, el cual carece necesariamente de presupuestos. Estos son los motivos que se encontraban detrás de una lectura «débil» de la $P h G$ (II y III.2).

Una posible solución a esta disyuntiva que quizá a alguien podría por de pronto ocurrírsele en un intento por conciliar una y otra alternativa es la siguiente. La $P h G$ no puede ser abandonada sin más, sino que debe ser mantenida, pero no como primera parte, sino como introducción, eso sí, como una introducción que no es meramente accidental o accesoria, puesto que, por lo menos, tiene un valor didáctico o propedéutico. Ahora bien, la pregunta fundamental es (o sigue siendo): ¿Cuál es el estatuto de esta introducción necesaria? ¿Tiene algún valor científico? Si no es la primera parte del «Sistema de la ciencia», ¿cómo otorgarle un valor que no sea meramente «histórico y raciocinante [räsonnierend]», esto es, accidental, dada la noción hegeliana de ciencia filosófica como sistema en sentido fuerte? La $P h G$, o bien es científica, o bien es sencillamente acientífica. Si la $P h G$ es acientífica, entonces no puede garantizar ni que las figuras de conciencia analizadas sean todas las figuras de conciencia posibles (con lo cual cabría la posibilidad de que alguna otra figura distinta del saber absoluto resultase no ser internamente contradictoria) ni que el encadenamiento de las figuras conduzca necesariamente al saber absoluto (ya que dichas figuras estarían agrupadas más o menos arbitrariamente sin orden

\footnotetext{
56 En favor de una lectura «fuerte» de la $P h G$, podemos preguntar de nuevo: ¿Pero por qué deberían estarlo? ¿Se le puede exigir al lector estar ya de entrada instalado en la perspectiva del saber absoluto? El propio Hegel parece responder negativamente a esta pregunta cuando, en el párrafo 26 del prólogo a la $P h G$, asegura que «el individuo tiene el derecho de que la ciencia le preste por lo menos una escalera para subir a ese punto de vista [al punto de vista de la ciencia]» (Hegel, G. W. F., Fenomenología del espíritu; op. cit., p. 130; Gesammelte Werke, Band 9, p. 23).
} 
interno alguno). Si, por el contrario, la $P h G$ es científica, entonces parece que lo es (si atendemos a las fragmentos citados en III.2) sólo por la Lógica a ella subyacente, de manera que la $P h G$ misma resulta que es, o bien, en efecto, redundante (y por tanto superflua), o bien simplemente ininteligible.

La tensión entre las posiciones presentadas en I y II respectivamente es una tensión inherente al concepto hegeliano de ciencia ${ }^{57}$ y que pone de manifiesto las dificultades que hay en la pretensión de que la filosofía, como «Sistema de la ciencia», consista en la presentación de lo absoluto. Más aún, dado que se pueden ofrecer, siguiendo a Hegel, argumentos prima facie igualmente buenos en favor tanto de una como de otra alternativa, el problema parece ser irresoluble desde dentro del propio Sistema hegeliano. Una introducción al saber absoluto es, por un lado, necesaria, pero, por otro y a la vez, imposible. La situación resulta, pues, hegelianamente aporética.

\section{Bibliografía}

\section{Fuentes}

Hegel, G. W. F., Ciencia de la lógica. Tomo I; Trad. A. y R. Mondolfo, Buenos Aires, Solar/Hachette, 19743 (Gesammelte Werke, Band 21: Wissenschaft der Logik. Die Lehre vom Sein. 1832; Hrsg. von F. Hogemann und W. Jaeschke, Hamburg, Felix Meiner, 1985).

\footnotetext{
57 La existencia de una tensión interna entre el Hegel de la $P h G$ y el Hegel maduro de la $W d L$ y la Enz no es, sin embargo, aceptada por todos los intérpretes de Hegel y la $P h G$. Forster, por ejemplo, no la acepta. Cf. sobre todo: Forster, M., Hegel's idea of a Phenomenology of spirit; op. cit., pp. 282-288 y 547-570. Según Forster, si los textos de Hegel son leídos correctamente, esa tensión resulta ser ficticia. La aparente incompatibilidad entre (i) la necesidad de una justificación del punto de vista del saber absoluto $(P h G)$ y (ii) la ausencia de presupuestos que se exige del comienzo lógico se resuelve (o, mejor dicho, se disuelve) si se atiende al hecho de que entre la $P h G$ y la $W d L$ tiene lugar una Aufhebung en el doble sentido de abolición y a la vez conservación. (Uno de los textos que Forster aduce en apoyo a su postura corresponde a la nota al parágrafo 12 de la Enz. En ella, Hegel dice que «el saber acerca de Dios, como el de todo lo suprasensible en general, contiene una elevación [Erhebung] sobre la sensación e intuición; contiene, por tanto, un comportamiento negativo respecto de eso primero y contiene por ende la mediación. Pues mediación es un comenzar y un haber avanzado hacia algo segundo, de modo que ese segundo sólo se da en tanto que se ha llegado a él desde otra cosa a él opuesta. Sin embargo, el saber acerca de Dios, opuesto a aquel lado empírico, no es por eso menos autosuficiente; es más, se da su propia autosuficiencia [Selbstständigkeit] esencialmente mediante esa negación y elevación». Hegel, G. W. F., Enciclopedia de las ciencias filosóficas en compendio, op. cit., pp. 114-115; Gesammelte Werke, Band 20, p. 53). Ahora bien, no está claro que la Aufhebung que tiene lugar entre la $P h G$ y la $W d L$ sea la solución al problema, aparente según Forster, y no más bien un particular modo de expresar o llamar la atención sobre el problema mismo de la relación entre la $P h G$ y la $W d L$.
} 
Hegel, G. W. F., Enciclopedia de las ciencias filosóficas en compendio; Trad. R. Valls Plana, Madrid Alianza, $2005^{4}$ (Gesammelte Werke, Band 20: Enziklopädie der philosophischen Wissenschaften im Grundrisse. 1830; Hrsg. von W. Bonsiepen und H. C. Lucas, Hamburg, Felix Meiner, 1992).

Hegel, G. W. F., Fenomenología del espíritu; Trad. M. Jiménez Redondo, Valencia, Pre-textos, 2006 (Gesammelte Werke, Band 9: Phänomenologie des Geistes. 1807; Hrsg. von W. Bonsiepen und R. Heede, Hamburg, Felix Meiner, 1980).

\section{Literatura secundaria}

Beiser, F. (ed.), The Cambridge companion to Hegel; Cambridge, Cambridge University Press, 1993.

Forster, M., Hegel's idea of a Phenomenology of spirit; Chicago, The University of Chicago Press, 1998.

Fulda, H. F., Das Problem einer Einleitung in Hegels Wissenschaft der Logik; Frankfurt am Main, Vittorio Klostermann, 1965.

Fulda, H. F., Henrich, D. (eds.), Materialien zu Hegels «Phänomenologie des Geistes»; Frankfurt am Main, Suhrkamp, 1975.

Habermas, J., Conocimiento e interés; Trad. M. Jiménez, J. F. Ivars y L. M. Santos, Madrid, Taurus, 1982 (Erkenntnis und Interesse; Frankfurt am Main, Suhrkamp, 1968).

Heidegger, M., Caminos de bosque; Trad. H. Cortés y A. Leyte, Madrid, Alianza, 2005 (Gesamtausgabe, Band 5: Holzwege; Frankfurt am Main, Vittorio Klostermann, 1977).

Henrich, D., Hegel en su contexto; Trad. J. A. Díaz, Caracas, Monte Ávila Editores, 1990 (Hegel im Kontext; Frankfurt am Main, Suhrkamp, 1971).

Martínez MarzoA, F., De Kant a Hölderlin; Madrid, Visor, 1992.

Martínez MarzoA, F., Hölderlin y la lógica hegeliana; Madrid, Visor, 1995.

Moyar, D., Quante, M., (eds.), Hegel's Phenomenology of spirit: A critical guide; Cambridge, Cambridge University Press, 2008.

Valls Plana, R., Del yo al nosotros. Lectura de la Fenomenología del espiritu de Hegel; Barcelona, PPU, 19943.

Nicolás García Mills

Facultad de Filosofía

Universidad de Barcelona

ngmills@hotmail.com 